Supplementary Information for:

\title{
Combined In-Situ Photoluminescence and X-ray Scattering Reveals Defect Formation in Lead-Halide Perovskite Films
}

Nada Mrkyvkova, ${ }^{1,2, *, \dagger}$ Vladimír Held, ${ }^{1, \dagger}$ Peter Nádaždy, ${ }^{1}$ Riyas Subair, ${ }^{1}$ Eva Majkova, ${ }^{1,2}$ Matej Jergel, ${ }^{1,2} \quad$ Aleš Vlk, ${ }^{3} \quad$ Martin Ledinsky, ${ }^{3} \quad$ Mário Kotlár ${ }^{4} \quad$ Jianjun Tian, ${ }^{5}$ and Peter Siffalovic ${ }^{1,2}$

${ }^{1}$ Institute of Physics, Slovak Academy of Sciences, Dúbravská cesta 9, 84511 Bratislava, Slovakia

${ }^{2}$ Center for Advanced Materials Application, Slovak Academy of Sciences, Dúbravská cesta 9, 84511 Bratislava, Slovakia

${ }^{3}$ Laboratory of Thin Films, Institute of Physics, ASCR, Cukrovarnická 10, 16200 Prague, Czech Republic

${ }^{4}$ Center for Nano-diagnostics, Slovak University of Technology, Vazovova 5, 81243 Bratislava, Slovakia

${ }^{5}$ Institute for Advanced Materials and Technology, University of Science and Technology Beijing, 100083 Beijing, China

${ }^{*}$ Corresponding author: nada.mrkyvkova@savba.sk

†Both authors contributed equally

Contents

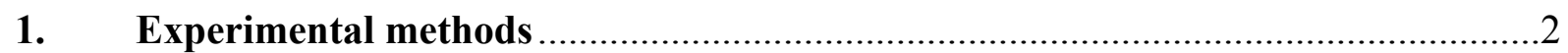

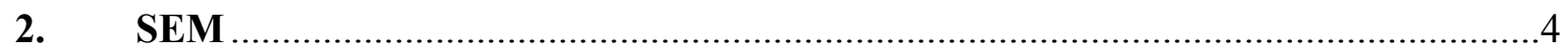

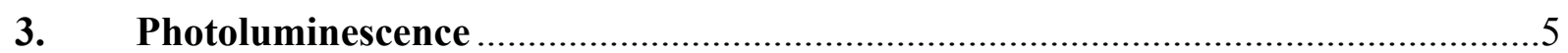

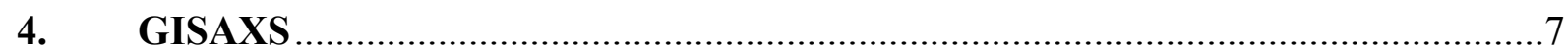

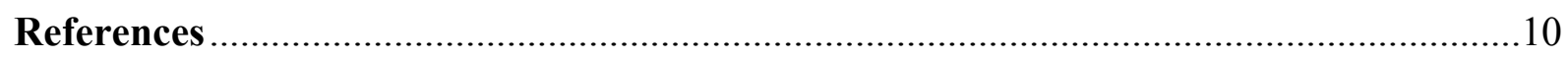




\section{Experimental methods}

\section{Perovskite film fabrication:}

$\mathrm{CH}_{3} \mathrm{NH}_{3} \mathrm{PbI}_{3-\mathrm{x}} \mathrm{Cl}_{\mathrm{x}}$ perovskite films were synthesized via a so-called single-step solution process. The $\mathrm{PbCl}_{2}, \mathrm{PbI}_{2}$ and methylammonium iodide (MAI) precursors, at a molar ratio of 1:1:4 ( $\left.\mathrm{PbCl}_{2}: \mathrm{PbI}_{2}: \mathrm{MAI}\right)$, dissolved in dimethylformamide (DMF) were purchased from Ossila (Perovskite Ink I201). The solution was spin-coated on a Si substrate with native oxide at $4000 \mathrm{rpm}$ for $30 \mathrm{~s}$. The samples were immediately transferred to the preheated plate for annealing $(t=0)$. During annealing, the samples were exposed to a nitrogen atmosphere to prevent any detrimental effects caused by air. $\mathrm{Si} / \mathrm{SiO}_{2}$ substrates (RMS roughness $\sim 0.4 \mathrm{~nm}$ ) were used to minimize X-ray surface scattering, especially in the GIWAXS measurements.

GIWAXS: The in-situ GIWAXS measurements were performed in the laboratory using MetalJet X-ray source from Excillum. The X-ray energy was $9.25 \mathrm{keV}$ and the total beam flux was $\sim 10^{8}$ photons/s. A hot plate (with the sample) was mounted on a hexapod motion platform, providing the tilt of the sample. The angle of incidence was set to $0.3^{\circ}$. GIWAXS patterns were recorded by a two-dimensional X-ray detector (Pilatus 300K, Dectris) with a $320 \mu \mathrm{m}$ thick Si sensor, enabling high temporal resolution.

Photoluminescence: The in-situ photoluminescence (PL) was measured in a custom-built setup comprising an excitation laser and a fiber-coupled spectrophotometer. The laser wavelength was $355 \mathrm{~nm}$ with an intensity of $\sim 1 \mathrm{~mW} / \mathrm{cm}^{2}$. The high excitation energy (in the UV spectral range) was used to increase the absorption and thus the PL intensity of the perovskite film. The PL spectra were measured in a time interval of 10 seconds.

The schematic sketch showing the simultaneous measurement of PL and GIWAXS during $\mathrm{MAPbI}_{3-\mathrm{x}} \mathrm{Cl}_{\mathrm{x}}$ perovskite formation is shown in Fig. $\mathrm{S} 1$. 


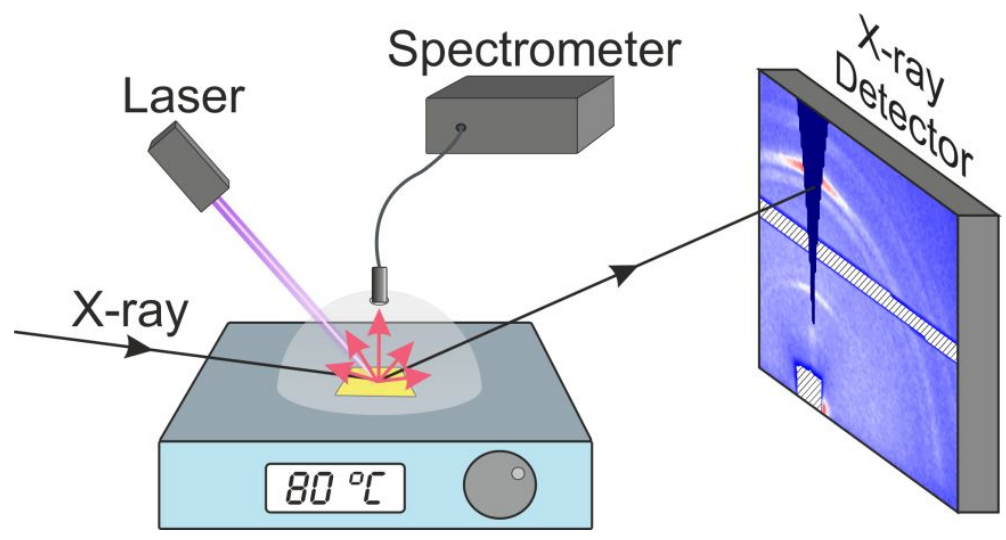

Figure S1. Schematic illustration of real-time GIWAXS and PL monitoring during the $\mathrm{MAPbI}_{3-\mathrm{x}} \mathrm{Cl}_{\mathrm{x}}$ perovskite crystallization. 


\section{2. $\underline{\text { SEM }}$}

The surface morphology of the perovskite layer was determined by scanning electron microscopy (SEM) after $\sim 60$ minutes of thermal annealing at $80{ }^{\circ} \mathrm{C}$ (see Fig. S2). The time interval of 60 minutes on the hot plate guarantees a dry surface without solvent residues that would modify the properties of the perovskite surface. SEM images confirm that a continuous layer is formed at such annealing times. Figure S2 shows a spot on the sample measured at three different magnifications.
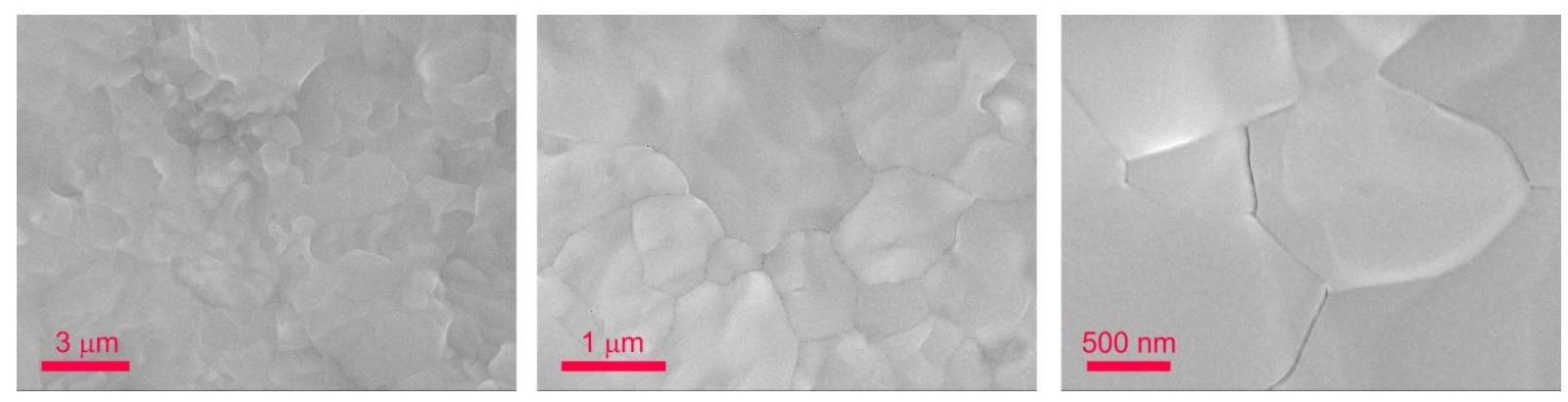

Figure S2. SEM images of the perovskite layer after thermal annealing at $80{ }^{\circ} \mathrm{C}$ for 60 minutes. The images were taken at different locations with various spatial resolutions. 


\section{Photoluminescence}

Here we show the photoluminescence (PL) dependence for the two chosen annealing temperatures $T=60{ }^{\circ} \mathrm{C}$ and $T=80{ }^{\circ} \mathrm{C}$. As mentioned in the main text, the measured PL spectra were fitted by the PseudoVoigt function (which well captured its asymmetrical shape in the early growth phase) to determine the Urbach energy, a spectral position of PL peak and its full width at half maximum (FWHM) (see Figs. S3, S4 and S5).

Based on the reciprocity relation between the PL and absorption spectrum, the absorptance spectrum was calculated using the generalized Planck's law (see Eq. S.1) for a photon current density $d j_{e}(h v)$ per energy interval.

$$
d j_{e}(h v) \approx A_{B B}(h v) \cdot(h v)^{2} \cdot e^{-\left(\frac{h v}{k_{B} T}\right)},
$$

where $h v$ is the photon energy, $A_{B B}(h v)$ is the band-to-band absorptance (being the function of $v$ ), $k_{B}$ is the Boltzmann constant, and $T$ is the absolute temperature. ${ }^{1}$ The energy dependence of absorptance in the logarithmic scale is shown in Fig. S3 (at different stages of annealing at $60^{\circ} \mathrm{C}$ ).

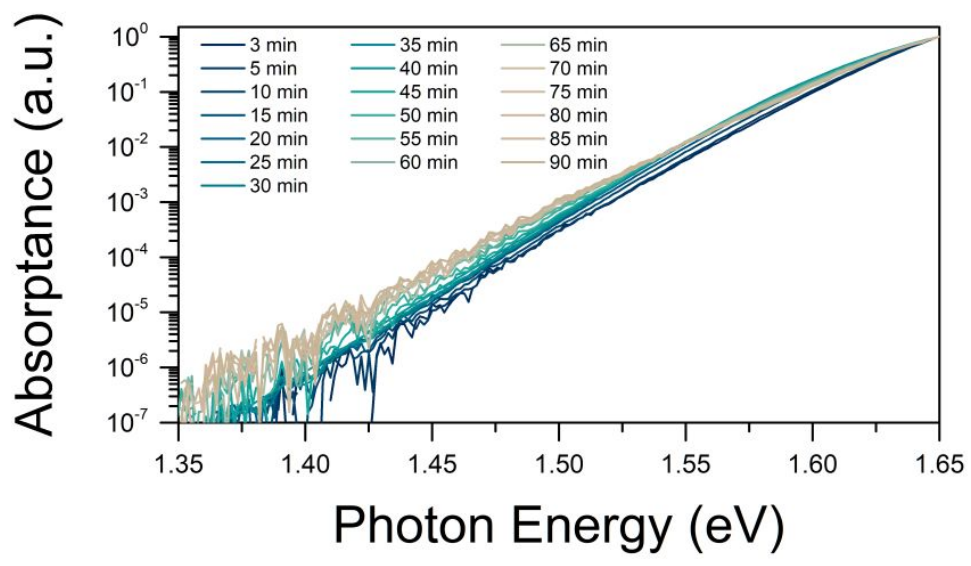

Figure S3. Normalized absorptance obtained from PL spectra during perovskite annealing at $60{ }^{\circ} \mathrm{C}$.

The Urbach energy $E_{U}(T)$ describes the slope of the absorption edge. To obtain its value, the absorptance spectra were normalized at $1.65 \mathrm{eV}$ and fitted by the so-called Urbach's rule ${ }^{2}$ (Eq. S.2) in the absorptance range of 0.004 to $8 \times 10^{-5}$. 


$$
\ln \left(A_{B B}(h v, T)\right)=\ln \left(A_{B B}\right)+h v_{E_{U}(T)}
$$

The time dependence of the Urbach energy and FWHM for both $T=60{ }^{\circ} \mathrm{C}$ and $T=80{ }^{\circ} \mathrm{C}$ are in Fig. S4 and Fig. S5, respectively.

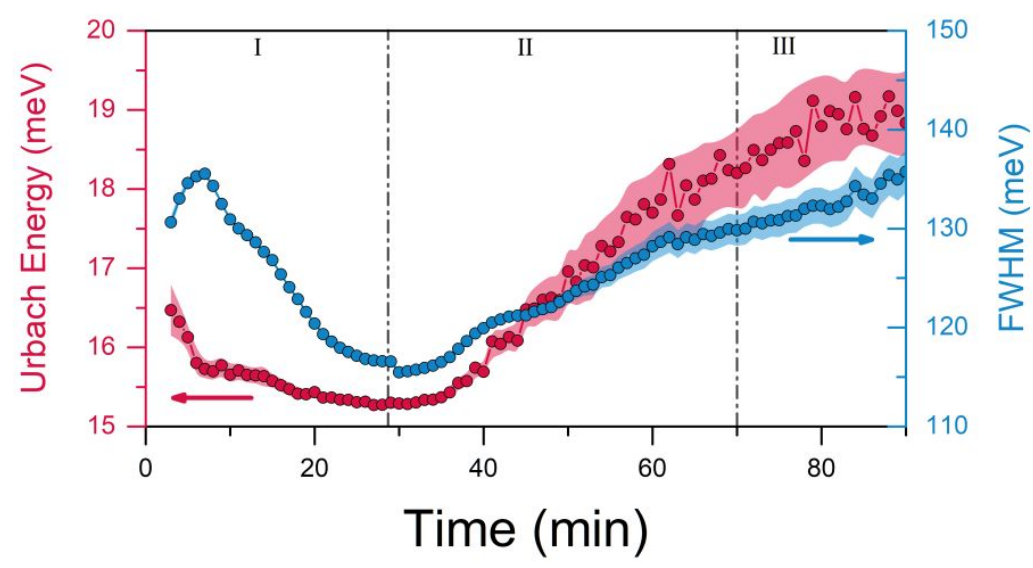

Figure S4. Urbach energy and FWHM of PL peaks obtained at the annealing temperature of $60{ }^{\circ} \mathrm{C}$.

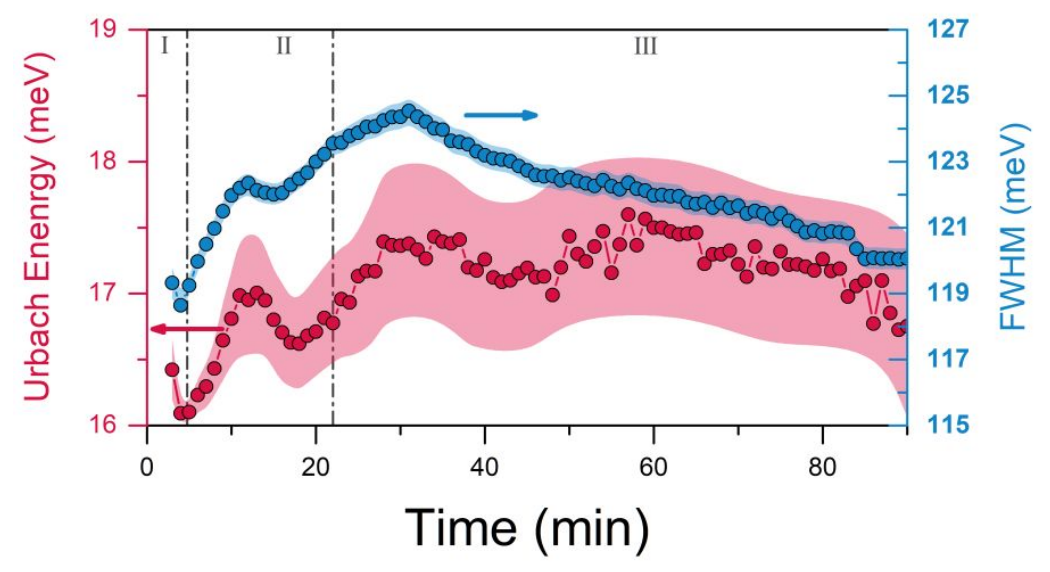

Figure S5. Urbach energy and FWHM of PL peaks obtained at the annealing temperature of $80{ }^{\circ} \mathrm{C}$. 


\section{GISAXS}

Apart from the standard grazing-incidence wide-angle X-ray scattering (GIWAXS) measurements, schematically shown in Fig. S1, we also performed simultaneous grazingincidence small-angle X-ray scattering (GISAXS) measurements during perovskite formation at the annealing temperature of $T=80^{\circ} \mathrm{C}$. GISAXS characterizes sample properties at low reciprocal $q$-values, down to $10^{-3} \AA^{-1}$, corresponding to length scales up to few hundreds of nanometers. In addition, simultaneous GISAXS and GIWAXS measurements provide information on film morphology and crystallization during the perovskite formation, respectively.

The configuration used is shown in Fig. S6. In this setup, the GIWAXS diffractions were detected by a one-dimensional detector (Dectris, Mythen), and the GISAXS patterns were detected by a two-dimensional detector (Dectris, Pilatus 300K).

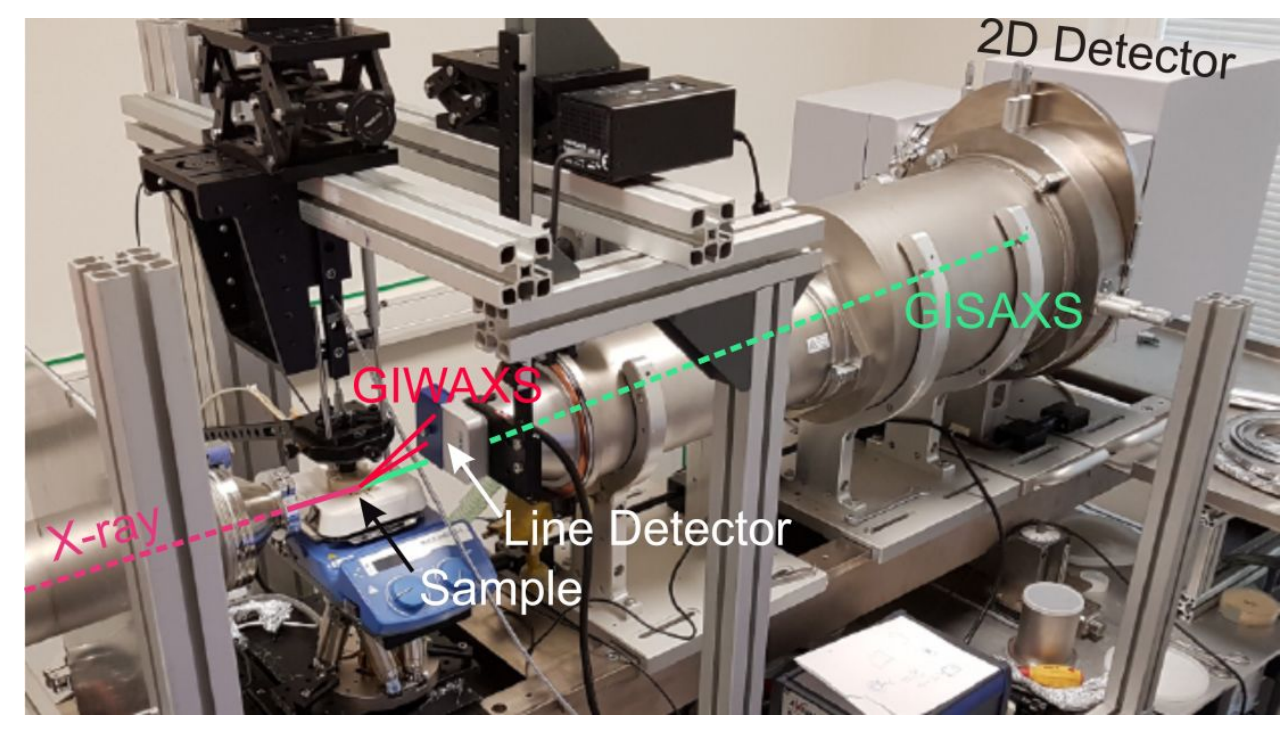

Figure S6. Experimental setup for simultaneous measurements of GIWAXS and GISAXS diffraction patterns.

Figure S7 shows the measured GISAXS pattern of the perovskite sample annealed for 30 minutes. The direct beam at $q=0 \AA^{-1}$ was blocked by a beam stop. The peaks at $q_{z} \sim 0.024 \AA^{-1}$ and $q_{z} \sim 0.05 \AA^{-1}$ correspond to the Yoneda peak and the specularly reflected beam, respectively. ${ }^{3}$ The GISAXS pattern was analyzed by performing horizontal line cuts in the Yoneda region on the right-hand side of the $q_{x y}$ range from 0 to $0.1 \AA^{-1}$. This interval was 
chosen because of the beamstop shading at the left-hand side of the $q$-range in Fig. S7. Such a 1D GISAXS profile (extracted from the 2D GISAXS pattern) is shown in Fig. S8.

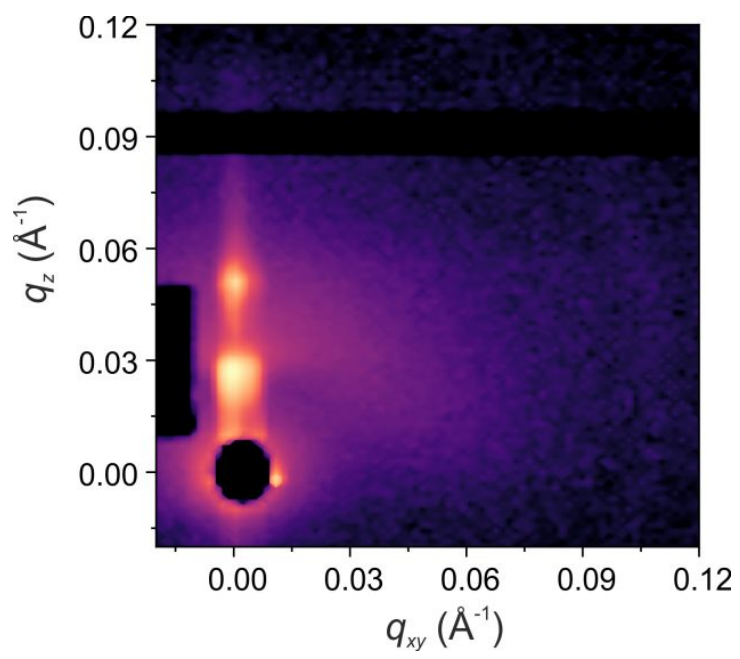

Figure S7. GISAXS reciprocal space map measured after $30 \mathrm{~min}$ of annealing at $80{ }^{\circ} \mathrm{C}$. The intensity increases from black to yellow.

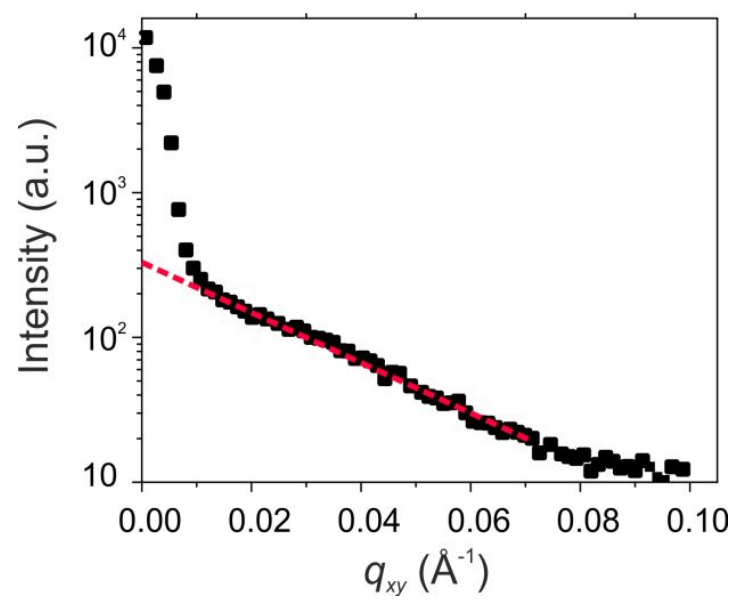

Figure S8. Horizontal linecut taken around Yoneda peak from the 2D GISAXS data measured after $30 \mathrm{~min}$ of the annealing at $80{ }^{\circ} \mathrm{C}$. The red dashed line indicates the experimental data fit in the range of $q_{x y}$ from 0.01 to $0.07 \AA^{-1}$.

Figure S8 shows that the scattered X-ray beam intensity increases dramatically for $q_{x y}<0.01 \AA^{-1}$ due to the surface roughness and the finite size of the primary beam, which is directly reflected in a broad shoulder of the Yoneda peak. In order to extrapolate the intensity directly at $q_{x y}=0 \AA^{-1}$, we fitted the scattered data by a linear function in the range of $q_{x y}$ from 
0.01 to $0.07 \AA^{-1}$. The obtained GISAXS intensity evolution at $q_{x y}=0 \AA^{-1}$ during the perovskite formation is shown in Fig. S9.

The intensity of X-ray scattering close to the origin of the reciprocal space (at $q_{z}$ of Yoneda peak and $q_{x y}=0 \AA^{-1}$ in our case) depends mainly on the electron density of the material. ${ }^{4}$ The GISAXS intensity evolution thus provides information about the solidification (freezing the electron density) of the sample. Figure S9 shows that the GISAXS intensity increases up to $\sim 40 \mathrm{~min}$ and does not change significantly thereafter. However, the $\mathrm{PbI}_{2}$ crystallites are being formed after 20 minutes of annealing. Thus, the GISAXS data suggest that the interface solidifies continuously at least 20 minutes after the sample was placed on the annealing plate. After 20 minutes, the $\mathrm{PbI}_{2}$ crystallites are formed (see Fig. S9) and might influence the GISAXS signal.

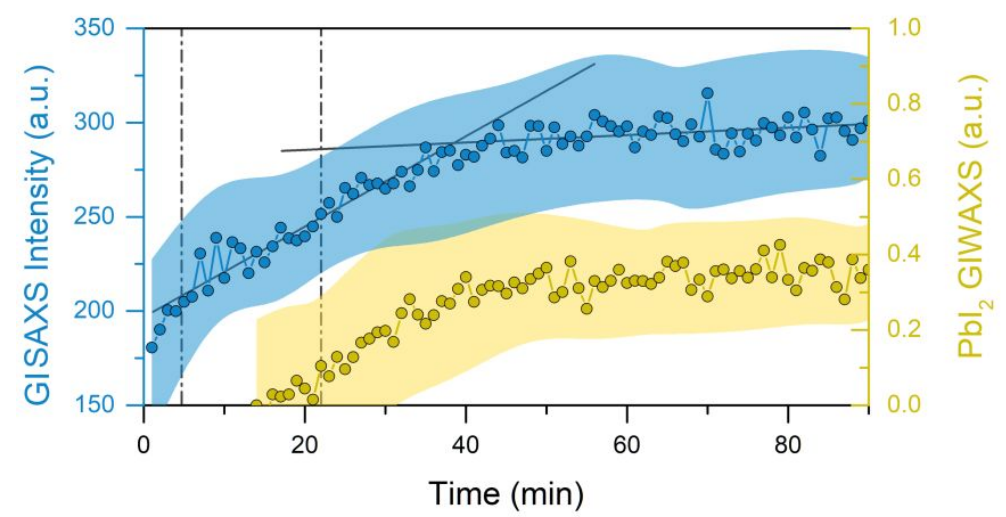

Figure S9. GISAXS intensity together with $\mathrm{PbI}_{2}$-phase intensity determined from GIWAXS measurements. 


\section{References}

(1) Barugkin, C.; Cong, J.; Duong, T.; Rahman, S.; Nguyen, H. T.; Macdonald, D.; White, T. P.; Catchpole, K. R. Ultralow Absorption Coefficient and Temperature Dependence of Radiative Recombination of $\mathrm{CH} 3 \mathrm{NH} 3 \mathrm{PbI} 3$ Perovskite from Photoluminescence. Journal of Physical Chemistry Letters 2015, 6 (5), 767-772. https://doi.org/10.1021/acs.jpclett.5b00044.

(2) Studenyak, I.; Kranjec, M.; Kurik, M. Urbach Rule in Solid State Physics. International Journal of Optics and Applications 2014, 4 (3), 76-83. https://doi.org/10.5923/j.optics.20140403.02.

(3) Renaud, G.; Lazzari, R.; Leroy, F. Probing Surface and Interface Morphology with Grazing Incidence Small Angle X-Ray Scattering. Surface Science Reports 2009, 64, 255-380. https://doi.org/doi:10.1016/j.surfrep.2009.07.002.

(4) Holý, V.; Pietsch, U.; Baumbach, T. High-Resolution X-Ray Scattering from Thin Films and Multilayers; Springer, 1999. 